\title{
From Pre-historic to Post-modern Symbolic Model Checking ${ }^{\star}$
}

\author{
Thomas A. Henzinger Orna Kupferman Shaz Qadeer \\ Department of EECS, University of California at Berkeley, CA 94720-1770, USA \\ Email: $\{$ tah, orna, shaz\}@eecs.berkeley.edu
}

\begin{abstract}
Symbolic model checking, which enables the automatic verification of large systems, proceeds by calculating with expressions that represent state sets. Traditionally, symbolic model-checking tools are based on backward state traversal; their basic operation is the function pre, which given a set of states, returns the set of all predecessor states. This is because specifiers usually employ formalisms with future-time modalities, which are naturally evaluated by iterating applications of pre. It has been recently shown experimentally that symbolic model checking can perform significantly better if it is based, instead, on forward state traversal; in this case, the basic operation is the function post, which given a set of states, returns the set of all successor states. This is because forward state traversal can ensure that only those parts of the state space are explored which are reachable from an initial state and relevant for satisfaction or violation of the specification; that is, errors can be detected as soon as possible.

In this paper, we investigate which specifications can be checked by symbolic forward state traversal. We formulate the problems of symbolic backward and forward model checking by means of two $\mu$-calculi. The pre- $\mu$ calculus is based on the pre operation; the post- $\mu$ calculus, on the post operation. These two $\mu$-calculi induce query logics, which augment fixpoint expressions with a boolean emptiness query. Using query logics, we are able to relate and compare the symbolic backward and forward approaches. In particular, we prove that all $\omega$-regular (linear-time) specifications can be expressed as post- $\mu$ queries, and therefore checked using symbolic forward state traversal. On the other hand, we show that there are simple branching-time specifications that cannot be checked in this way.
\end{abstract}

\section{Introduction}

Today's rapid development of complex and safety-critical systems requires verifi cation methods such as model checking. In model checking [CE81,QS81], we ensure that a system exhibits a desired behavior by executing an algorithm that checks whether a mathematical model of the system satisfi es a formal specification that describes the behavior. The algorithmic nature of model checking makes it fully automatic, and thus attractive to practitioners. At the same time, model checking is very sensitive to the size of the mathematical model of the system. Commercial model-checking tools need to cope with the exceedingly large state spaces that are present in reallife examples, making the so-called state-explosion problem perhaps the most challenging issue in computer-aided verifi cation. One of the important developments in this area is the discovery of symbolic model-checking methods [BCM ${ }^{+}$92]. In particular, use of BDDs [Bry86] for model representation has yielded model-checking tools that can handle very large state spaces [CGL93].

Traditional symbolic model-checking tools have been based on backward state traversal $\left[\mathrm{McM}^{2}, \mathrm{BHSV}^{+} 96\right]$. They compute with expressions that represent state sets using, in addition to positive boolean operations, the functions pre and $\widetilde{p r e}$, which map a set of states to a subset

\footnotetext{
* This work is supported in part by ONR YIP award N00014-95-1-0520, by NSF CAREER award CCR9501708, by NSF grant CCR-9504469, by ARO MURI grant DAAH-04-96-1-0341, and by the SRC contract 97-DC-324.041.
} 
of its predecessor states. Formally, given a set $U$ of states, the set pre $(U)$ contains the states for which there exists a successor state in $U$, and the set $\widetilde{p r e}(U)$ contains the states all of whose successor states are in $U$. By evaluating fi xpoint expressions over boolean and pre operations, complicated state sets can be calculated. For example, to fi nd the set of states from which a state satisfying a predicate $p$ is reachable, the model checker starts with the set $U$ of states in which $p$ holds, and repeatedly adds to $U$ the set $\operatorname{pre}(U)$, until no more states can be added. Formally, the model checker calculates the least fi xpoint of the expression $U=p \vee \operatorname{pre}(U)$. Symbolic modelchecking techniques were fi rst applied to branching-time specifi cations, and later extended to linear-time specifi cations, both via translations into fi xpoint expressions [BCM\$92,CGH94].

As an alternative to symbolic model checking, in enumerative model checking states are represented individually. Traditional enumerative model-checking tools check linear-time specifi cations by forward state traversal [Hol97,Di196]. There, the basic operation is to compute, for a given state, the list of successor states. Forward state traversal has several obvious advantages over backward state traversal. First, for operational system models, successor states are often easier to compute than predecessor states. Second, only the reachable part of the state space is traversed. Third, optimizations such as on-the-fly [GPVW95] and partial-order [Pe194] methods can be incorporated naturally. For example, in on-the-fly model checking, only those parts of the state space are traversed which are relevant for satisfying (or violating) the given specifi cation.

Some of the advantages of forward state traversal can be easily incorporated into symbolic methods. For example, we may fi rst compute the set of reachable states by symbolic forward state traversal, and then restrict backward state traversal for model checking to the reachable states. This method, however, is unsatisfactory; for example, it cannot fi nd even a short error trace if the set of reachable states cannot be computed. We present a tighter, and more advantageous, integration of forward state traversal with symbolic methods. In symbolic forward state traversal, we replace the functions pre and $\widetilde{p r e}$ by the functions post and $\widetilde{\text { post}}$, respectively, which map a set of states to a subset of its successor states. Formally, given a set $U$ of states, the set $\operatorname{post}(U)$ contains the states for which there exists a predecessor state in $U$, and the set $\widetilde{p o s t}(U)$ contains the states all of whose predecessor states are in $U$. Then, we evaluate fi xpoint expressions over boolean and post operations on state sets. It has recently been shown that certain branching-time as well as linear-time specifi cations, such as response (i.e., $\square(p \rightarrow \diamond q)$ ), can be model checked by symbolic forward state traversal [INH96,IN97]. We attempt a more systematic study of what can and what cannot be model checked in this way. In particular, we show that all $\omega$-regular (linear-time) specifi cations (which include all LTL specifi cations) are amenable to a symbolic forward approach, while some CTL (branching-time) specifi cations are not.

For this purpose, we defi ne post- $\mu$, a fi xpoint calculus that is based on post operations in the same way in which the traditional $\mu$-calculus, here called $p r e-\mu$, is based on pre operations [Koz83]. While pre- $\mu$ expressions refer to the future of a given state in a model, post- $\mu$ expressions refer to its past. Therefore, in stark contrast to the fact that every LTL and CTL specifi cation has an equivalent expression in pre- $\mu$, almost no LTL or CTL specifi cation, including response, has an equivalent expression in post- $\mu$. In order to compare pre and post logics, rather, we need to defi ne query logics, whose formulas refer to a whole model, not an individual state. Query logics are based on the emptiness predicate $\mathcal{E}$. For a specifi cation $\phi$, which is true in some states of a model and false in others, the query $\mathcal{E}(\phi)$ is true in a model iff $\phi$ is false in all states of the model. The query logic post- $\mu_{\emptyset}$ contains all queries of the form $\mathcal{E}(\phi)$ and $\neg \mathcal{E}(\phi)$, for post- $\mu$ expressions $\phi$. On the positive side, we prove that every $\omega$-regular (Büchi) specifi cation has an equivalent query in post- $\mu_{\emptyset}$. As with the translation from Büchi automata to pre- $\mu$ expressions [EL86,BC96], the translation from Büchi automata to post- $\mu_{\emptyset}$ queries is linear and involves only fi xpoint expressions of alternation depth two. Moreover, we show that every co-Büchi specifi cation has an equivalent query in alternation-free post- $\mu$, which can be checked effi ciently (in linear time). On the negative side, we prove that there are CTL specifi cations that are not equivalent to any boolean combination of post- $\mu_{\emptyset}$ queries. 
Symbolic forward model checking combines the benefi ts of symbolic over enumerative state traversal with the benefi ts of forward over backward state traversal. In [INH96,IN97], the authors present experimental evidence that symbolic forward state traversal can be signifi cantly more effi cient than symbolic backward state traversal. Our preliminary experimental results confi rm this observation. In addition, we give some theoretical justifi cations for the symbolic forward approach. We show that unlike enumerative forward model checking (which is traditionally based on depth-fi rst state traversal) and unlike symbolic backward model checking, the symbolic forward approach guarantees a.s.a.p. error detection. Intuitively, if a model violates a safety specifi cation, and the shortest error trace has length $m$, then the breadth-fi rst nature of symbolic forward model checking ensures that the error will be found before any states at a distance greater than $m$ from the initial states are explored.

The remainder of this paper is organized as follows. In Section 2 we defi ne the logics pre- $\mu$ and post- $\mu$, and the query logics they induce. In Section 3, we translate Büchi automata into equivalent post- $\mu_{\emptyset}$ queries of alternation depth two, and co-Büchi automata into equivalent alternation-free post- $\mu_{\emptyset}$ queries. We also show that the translation guarantees a.s.a.p. error detection for safety specifi cations. In Section 4, we compare the distinguishing and expressive powers of the various pre, post, and query logics. Finally, in Section 5 we put our results in perspective and report on some experimental evidence for the value of symbolic forward model checking.

\section{Definition of Pre and Post Logics}

\subsection{Pre and post $\mu$-calculi}

The $\mu$-calculus is a modal logic augmented with least and greatest fi xpoint operators [Koz83]. In this paper, we use the equational form of the propositional $\mu$-calculus, as in [BC96]. The modalities of the $\mu$-calculus relate a set of states to a subset of its predecessor states. Therefore, we refer to the $\mu$-calculus by $p r e-\mu$.

The formulas of pre- $\mu$ are defi ned with respect to a set $P$ of propositions and a set $V$ of variables. A modal expression is either $p, \neg p, X, \varphi \vee \psi, \varphi \wedge \psi, \exists \bigcirc \varphi$, or $\forall \bigcirc \varphi$, for propositions $p \in P$, variables $X \in V$, and modal expressions $\varphi$ and $\psi$. Let $I$ be a fi nite subset of the set of natural numbers. An equational block $B=\left\langle\lambda,\left\{X_{i}=\varphi_{i} \mid i \in I\right\}\right\rangle$ consists of a flag $\lambda \in\{\mu, \nu\}$ and a fi nite set of equations $X_{i}=\varphi_{i}$, where each $X_{i}$ is a variable, each $\varphi_{i}$ is a modal expression, and the variables $X_{i}$ are pairwise distinct. If $\lambda=\mu$, then $B$ is a $\mu$-block; otherwise $B$ is a $\nu$-block. For the equational block $B$, let $\operatorname{vars}(B)=\left\{X_{i} \mid i \in I\right\}$ be the set of variables on the left-hand sides of the equations of $B$. A block tuple $\mathcal{B}=\left\langle B_{1}, \ldots, B_{n}\right\rangle$ is a fi nite list of equational blocks such that the variable sets $\operatorname{vars}\left(B_{j}\right)$, for $1 \leq j \leq n$, are pairwise disjoint. For the block tuple $\mathcal{B}$, let $\operatorname{vars}(\mathcal{B})=\bigcup_{1 \leq j \leq n} \operatorname{vars}\left(B_{j}\right)$. For every variable $X \in \operatorname{vars}(\mathcal{B})$, let expand $_{\mathcal{B}}(X)$ be the modal expression on the right-hand side of the unique equation in $\mathcal{B}$ whose left-hand side is $X$. A pre- $\mu$ formula $\phi=\left\langle\mathcal{B}, X_{0}\right\rangle$ is a pair that consists of a block tuple $\mathcal{B}$ and a variable $X_{0} \in \operatorname{vars}(\mathcal{B})$. The variable $X_{0}$ is called the root variable of $\phi$. The formula $\phi$ is a pre- $\mu$ sentence if every variable that occurs in some modal expression of $\mathcal{B}$ is contained in $\operatorname{vars}(\mathcal{B})$.

The semantics of a $p r e-\mu$ formula is defi ned with respect to a Kripke structure and a valuation for the variables. A Kripke structure is a tuple $K=\langle P, W, R, L\rangle$ that consists of a fi nite set $P$ of propositions, a fi nite set $W$ of states, a binary transition relation $R \subseteq W \times W$ total in both the fi rst and second arguments (i.e., for every state $w \in W$, there is a state $w^{\prime}$ such that $R\left(w, w^{\prime}\right)$ and there is a state $w^{\prime \prime}$ such that $R\left(w^{\prime \prime}, w\right)$ ), and a labeling function $L: W \rightarrow 2^{P}$ that assigns to each state a set of propositions. The set $P$ of propositions contains the distinguished proposition init; a state $w \in W$ is initial if init $\in L(w)$. We defi ne four functions pre, $\widetilde{\text { pre }}$, post and $\widetilde{\text { post }}$ 
from $2^{W}$ to $2^{W}$ as follows. For any set $U \subseteq W$ of states, let

$$
\begin{aligned}
& \operatorname{pre}(U)=\left\{w \in W \mid \text { there exists a state } w^{\prime} \in U \text { with } R\left(w, w^{\prime}\right)\right\}, \\
& \widetilde{p r e}(U)=\left\{w \in W \mid \text { for all states } w^{\prime} \text { with } R\left(w, w^{\prime}\right), \text { we have } w^{\prime} \in U\right\}, \\
& \operatorname{post}(U)=\left\{w \in W \mid \text { there exists a state } w^{\prime} \in U \text { with } R\left(w^{\prime}, w\right)\right\}, \\
& \widetilde{\operatorname{post}}(U)=\left\{w \in W \mid \text { for all states } w^{\prime} \text { with } R\left(w^{\prime}, w\right), \text { we have } w^{\prime} \in U\right\} .
\end{aligned}
$$

A $K$-valuation for a set $V$ of variables is a function $\Gamma: V \rightarrow 2^{W}$ that assigns to each variable a set of states. If $\Gamma$ and $\Gamma^{\prime}$ are $K$-valuations for $V$, and $V^{\prime} \subseteq V$ is a subset of the variables, we write $\Gamma\left[\Gamma^{\prime} / V^{\prime}\right]$ for the $K$-valuation for $V$ that assigns $\Gamma^{\prime}(\bar{X})$ to each variable $X \in V^{\prime}$, and $\Gamma(X)$ to each variable $X \in V \backslash V^{\prime}$.

Given a Kripke structure $K=\langle P, W, R, L\rangle$ and a $K$-valuation $\Gamma$ for a set $V$ of variables, every modal expression $\varphi$ over the propositions $P$ and the variables $V$ defi nes a set $\varphi^{K}(\Gamma) \subseteq W$ of states: inductively, $p^{K}(\Gamma)=\{w \in W \mid p \in L(w)\},(\neg p)^{K}(\Gamma)=\{w \in W \mid p \notin L(w)\}$, $X^{K}(\Gamma)=\Gamma(X),(\varphi \vee \psi)^{K}(\Gamma)=\varphi^{K}(\Gamma) \cup \psi^{K}(\Gamma),(\varphi \wedge \psi)^{K}(\Gamma)=\varphi^{K}(\Gamma) \cap \psi^{K}(\Gamma)$, $(\exists \bigcirc \varphi)^{K}(\Gamma)=\operatorname{pre}\left(\varphi^{K}(\Gamma)\right)$, and $(\forall \bigcirc \varphi)^{K}(\Gamma)=\widetilde{p r e}\left(\varphi^{K}(\Gamma)\right)$. Given $K$, every block tuple $\mathcal{B}=\left\langle B_{1}, \ldots, B_{n}\right\rangle$ over $P$ and $V$ defines a function $\mathcal{B}^{K}$ from the $K$-valuations for $V$ to the $K$-valuations for $V$ : inductively, if $n=0$, then $\mathcal{B}^{K}(\Gamma)=\Gamma$; if $B_{1}$ is a $\mu$-block, then $\mathcal{B}^{K}(\Gamma)$ is the least fi xpoint of the function $F_{\mathcal{B}, \Gamma}^{K}$; if $B_{1}$ is a $\nu$-block, then $\mathcal{B}^{K}(\Gamma)$ is the greatest fi xpoint of $F_{\mathcal{B}, \Gamma}^{K}$. The monotonic function $F_{\mathcal{B}, \Gamma}^{K}$ from valuations to valuations is defi ned by

$$
F_{\mathcal{B}, \Gamma}^{K}\left(\Gamma^{\prime}\right)(X)= \begin{cases}\operatorname{expand}(X)^{K}\left(\left\langle B_{2}, \ldots, B_{n}\right\rangle^{K}\left(\Gamma\left[\Gamma^{\prime} / \operatorname{vars}\left(B_{1}\right)\right]\right)\right) & \text { if } X \in \operatorname{vars}\left(B_{1}\right), \\ \left(\left\langle B_{2}, \ldots, B_{n}\right\rangle^{K}\left(\Gamma\left[\Gamma^{\prime} / \text { vars }\left(B_{1}\right)\right]\right)\right)(X) & \text { otherwise. }\end{cases}
$$

Note that for a pre- $\mu$ sentence $\phi=\left\langle\mathcal{B}, X_{0}\right\rangle$, the function $\mathcal{B}^{K}$ is a constant function. Given $K$, the sentence $\phi$ defi nes the set $\phi^{K}=\mathcal{B}^{K}(\Gamma)\left(X_{0}\right)$ of states (for any choice of $\Gamma$ ). For a state $w \in W$ and a pre- $\mu$ sentence $\phi$, we write $w \models_{K} \phi$ if $w \in \phi^{K}$. For a Kripke structure $K$, we write $K \models \phi$, and say that $K$ satisfies $\phi$, if there is an initial state $w$ of $K$ such that $w \models_{K} \phi .{ }^{1}$ The model-checking problem for pre- $\mu$ is to decide, given a Kripke structure $K$ and a pre- $\mu$ sentence $\phi$, whether $K=\phi$.

Given a block tuple $\mathcal{B}=\left\langle B_{1}, \ldots, B_{n}\right\rangle$, the block $B_{i}$ depends on the block $B_{j}$ if $i \neq j$ and some variable that occurs in a modal expression of $B_{i}$ is contained in vars $\left(B_{j}\right)$. The pre- $\mu$ sentence $\phi=\left\langle\mathcal{B}, X_{0}\right\rangle$ is alternation-free if the dependency relation on the blocks of $\mathcal{B}$ is acyclic (i.e., its transitive closure is asymmetric). The model-checking problem for the alternation-free fragment of $p r e-\mu$ can be solved in linear time [CS91].

The logic post- $\mu$ is obtained from the logic pre- $\mu$ by replacing the future modal operators $\exists \bigcirc$ and $\forall \bigcirc$ by the past modal operators $\exists \odot$ and $\forall \odot$, with the interpretations $(\exists \odot \varphi)^{K}(\Gamma)=$ post $\left(\varphi^{K}(\Gamma)\right)$ and $(\forall \odot \varphi)^{K}(\Gamma)=\widetilde{p o s t}\left(\varphi^{K}(\Gamma)\right)$. The semantics of post- $\mu$ can alternatively be defi ned as follows. For a Kripke structure $K=\langle P, W, R, L\rangle$, defi ne the Kripke structure $K^{-1}=\left\langle P, W, R^{-1}, L\right\rangle$, where $R^{-1}\left(w, w^{\prime}\right)$ iff $R\left(w^{\prime}, w\right)$. For a post- $\mu$ sentence $\phi$, defi ne $\phi^{-1}$ to be the pre- $\mu$ sentence obtained from $\phi$ by replacing each occurrence of $\exists \odot$ and $\forall \odot$ by $\exists \bigcirc$ and $\forall \bigcirc$, respectively. Then, for every state $w$ of $K$, we have $w \models_{K} \phi$ iff $w \models_{K^{-1}} \phi^{-1}$.

\subsection{Query logics}

We defi ne query logics that are based on pre- $\mu$ and post- $\mu$. The sentences of pre- $\mu$ refer to the future of a given state in a Kripke structure, and the sentences of post- $\mu$ refer to its past. By contrast, the sentences of query logics, called queries, refer to the whole structure and thus enable us to translate between pre and post logics. The query logics are obtained from $p r e-\mu$ and

\footnotetext{
${ }^{1}$ Note that we work, for convenience, with the dual of the usual requirement that all initial states satisfy a pre- $\mu$ sentence.
} 
post- $\mu$ by adding a predicate $\mathcal{E}$ on sentences, called the emptiness predicate. For a logic $\mathcal{L}$, the query logic $\mathcal{L}_{\emptyset}$ contains the two queries $\mathcal{E}(\phi)$ and $\neg \mathcal{E}(\phi)$ for each sentence $\phi$ of $\mathcal{L}$. The query $\operatorname{logic} \mathcal{L}_{\mathcal{E}}$ is richer and its queries are constructed inductively as follows:

- $\mathcal{E}(\phi)$, where $\phi$ is a formula of $\mathcal{L}$,

- $\neg \theta_{1}$ and $\theta_{1} \vee \theta_{2}$, where $\theta_{1}$ and $\theta_{2}$ are queries of $\mathcal{L}_{\mathcal{E}}$.

The satisfaction relation $=$ for queries on a Kripke structure $K$ is inductively defi ned as follows:

- $K \models \mathcal{E}(\phi)$ iff for all states $s$ of $K$, we have $s \not \models \phi$,

- $K \models \neg \theta_{1}$ iff $K \mid \forall \theta_{1}$, and $K \models \theta_{1} \vee \theta_{2}$ iff $K \models \theta_{1}$ or $K \models \theta_{2}$.

While our motivation for query logics is theoretical, for the purpose of comparing pre and post logics, query logics are also practical. This is because once the state set $\phi^{K}$ has been computed (either explicitly or implicitly, using BDDs), the evaluation of the query $\mathcal{E}(\phi)$ requires constant time. Therefore, checking a query in $p r e-\mu_{\mathcal{E}}$ or $p o s t-\mu_{\mathcal{E}}$ is no harder than model checking pre- $\mu$ or post- $\mu$, respectively.

\subsection{Equivalences on Kripke structures induced by pre and post logics}

Let $K=\langle P, W, R, L\rangle$ and $K^{\prime}=\left\langle P, W^{\prime}, R^{\prime}, L^{\prime}\right\rangle$ be two Kripke structures with the same set of propositions. A relation $\beta \subseteq W \times W^{\prime}$ is a pre-bisimilarity relation if for all states $w$ and $w^{\prime}$, we have that $\beta\left(w, w^{\prime}\right)$ implies (1) $L(w)=L^{\prime}\left(w^{\prime}\right)$, (2) for every state $v$ with $R(w, v)$, there is a state $v^{\prime}$ with $R^{\prime}\left(w^{\prime}, v^{\prime}\right)$ and $\beta\left(v, v^{\prime}\right)$, and (3) for every state $v^{\prime}$ with $R^{\prime}\left(w^{\prime}, v^{\prime}\right)$, there is a state $v$ with $R(w, v)$ and $\beta\left(v, v^{\prime}\right)$. Note that, in particular, $\beta\left(w, w^{\prime}\right)$ implies that either both $w$ and $w^{\prime}$ are initial, or neither of them is initial. The pre-bisimilarity relation $\beta$ is a pre-bisimulation between $K$ and $K^{\prime}$ if for all states $w \in W$, there is a state $w^{\prime} \in W^{\prime}$ such that $\beta\left(w, w^{\prime}\right)$, and for all states $w^{\prime} \in W^{\prime}$, there is a state $w \in W$ such that $\beta\left(w, w^{\prime}\right)$. The pre-bisimilarity relation $\beta$ is an init-pre-bisimulation between $K$ and $K^{\prime}$ if for all initial states $w \in W$, there is an initial state $w^{\prime} \in W^{\prime}$ such that $\beta\left(w, w^{\prime}\right)$, and for all initial states $w^{\prime} \in W^{\prime}$, there is an initial state $w \in W$ such that $\beta\left(w, w^{\prime}\right)$. The relation $\beta \subseteq W \times W^{\prime}$ is a post-bisimulation (resp. init-postbisimulation) between $K$ and $K^{\prime}$ if $\beta$ is a pre-bisimulation (resp. init-pre-bisimulation) between $K^{-1}$ and $K^{\prime-1}$. The following is an easy extension of a well-known result for pre- $\mu$ [BCG88].

Proposition 1. Let $K$ and $K^{\prime}$ be two Kripke structures.

- There is an init-pre-bisimulation (resp. init-post-bisimulation) between $K$ and $K^{\prime}$ iff for all sentences $\phi$ of pre- $\mu$ (resp. post- $\mu$ ), we have $K=\phi$ iff $K^{\prime} \models \phi$.

- The following three statements are equivalent:

(1) There is a pre-bisimulation (resp. post-bisimulation) between $K$ and $K^{\prime}$.

(2) For all queries $\theta$ of pre- $\mu_{\emptyset}$ (resp. post- $\left.\mu_{\emptyset}\right)$, we have $K \models \theta$ iff $K^{\prime}=\theta$.

(3) For all queries $\theta$ of pre- $\mu_{\mathcal{E}}$ (resp. post- $\mu_{\mathcal{E}}$ ), we have $K=\theta$ iff $K^{\prime}=\theta$.

\section{Intersection of Pre and Post Logics}

Of particular interest is the intersection of the query logics pre- $\mu_{\mathcal{E}}$ and post- $\mu_{\mathcal{E}}$. It contains the queries that can be specifi ed in both pre- $\mu_{\varepsilon}$, which often is more convenient for specifi ers, and in post- $\mu_{\mathcal{E}}$, which often is more effi cient for symbolic model checking. In this section we show that essentially all linear properties lie in this intersection. On the other hand, there are simple branching properties that do not lie in the intersection. 


\subsection{In}

Consider a Kripke structure $K=\langle P, W, R, L\rangle$. An observation of $K$ is a subset of the propositions $P$. An error trace of $K$ is a fi nite or infi nite sequence of observations. A linear property of $K$ is a set of error traces. ${ }^{2}$ Many useful linear properties, namely, the $\omega$-regular linear properties, can be specifi ed by fi nite automata. A finite automaton $A=\left\langle P, S, S, S_{F}, r, \ell\right\rangle$ consists of a fi nite set $P$ of propositions, a fi nite set $S$ of states, a set $S \subseteq S$ of initial states, a set $S_{F} \subseteq S$ of accepting states, a binary transition relation $r \subseteq S \times S$, and a labeling function $\ell: S \rightarrow 2^{P}$ that assigns to each state a set of propositions. The following defi nitions regarding paths apply equally to Kripke structures and automata. A path $\pi=u_{0}, u_{1}, \ldots$ of $K$ (resp. $A$ ) is a fi nite or infi nite sequence of states such that for all $i \geq 0$, we have $R\left(u_{i}, u_{i+1}\right)$ (resp. $r\left(u_{i}, u_{i+1}\right)$ ). The path $\pi$ is initialized if $u_{0}$ is an initial state. By $\operatorname{Inf}(\pi)$ we denote the set of states that appear in $\pi$ infi nitely often. The labeling functions $L$ and $\ell$ are lifted from states to paths in the obvious way.

With each fi nite automaton $A$ we associate a sentence $\exists A$ that is interpreted over a Kripke structure $K$ with the same propositions as $A$. The model-checking problem for automata is to decide, given $K$ and $A$, whether $K \models \exists A$. We defi ne $K \models \exists A$ if there exist an initialized path $\pi_{1}$ of $K$ and an accepting initialized path $\pi_{2}$ of $A$ such that $L\left(\pi_{1}\right)=\ell\left(\pi_{2}\right)$; such an observation sequence $L\left(\pi_{1}\right)$ is called an error trace of $K$ with respect to $A$. Which paths of $A$ are accepting depends on the interpretation we place on the automaton $A$. We consider here three different interpretations: safety automata, Büchi automata, and co-Büchi automata. For each interpretation we reduce the model-checking problem for automata to the model-checking problem for post- $\mu_{\emptyset}$, by translating automata into equivalent post- $\mu_{\emptyset}$ queries. The post $\mu_{\emptyset}$ query $\theta$ is equivalent to the automaton $A$ if for every Kripke structure $K$, we have $K \models \exists A$ iff $K \models \theta$.

In all translations, we will make use of the following. With each state $s$ of the automaton $A$, we associate two variables, $X_{s}$ and $X_{s}^{\prime}$. In addition, we use the two variables $X_{F}$ and $X_{F}^{\prime}$. For each state $s$ of $A$, let $\gamma_{s}$ be a variable-free and modality-free expression that characterizes states locally, namely, $\gamma_{s}=\bigwedge_{p \in \ell(s)} p \wedge \bigwedge_{p \notin \ell(s)} \neg p$. Now, let $B_{A}$ be the following $\mu$-block, which consists of $|S|+1$ equations, with $\operatorname{vars}\left(B_{A}\right)=\left\{X_{s} \mid s \in S\right\} \cup\left\{X_{F}\right\}$ :

$$
\begin{aligned}
& X_{s}= \begin{cases}\gamma_{s} \wedge\left(\text { init } \vee \bigvee_{t \in \text { pre }(s)} \exists \odot X_{t}\right) & \text { if } s \in S_{0}, \\
\gamma_{s} \wedge \bigvee_{t \in \text { pre }(s)} \exists \odot X_{t} & \text { if } s \notin S_{0},\end{cases} \\
& X_{F}=\bigvee_{f \in S_{F}} X_{f} .
\end{aligned}
$$

Note that the size of $B_{A}$ is linear in the size of $A$.

Safety automata A safety property of a Kripke structure $K$ is a set of finite error traces. The regular safety properties can be specifi ed by safety automata. A safety automaton is a fi nite automaton $A$ such that a path $\pi$ of $A$ is accepting if $\pi$ is a fi nite path and its last state is an accepting state of $A$. It is not diffi cult to see that the safety automaton $A$ is equivalent to the post- $\mu_{\emptyset}$ query $\theta_{A}=\neg \mathcal{E}\left(\left\langle\left\langle B_{A}\right\rangle, X_{F}\right\rangle\right)$.

If a fi nite error trace exists, during model checking, we would like to find it as soon as possible. By evaluating the query $\theta_{A}$ as follows (in the standard way), this can indeed be guaranteed. The evaluation of the $\mu$-block $B_{A}$ over a Kripke structure $K$ proceeds in iterations. Let $X^{K}(i) \subseteq W$ denote the value of variable $X \in \operatorname{vars}\left(B_{A}\right)$ after the $i$-th iteration, and let $\Gamma^{K}(i)$ denote the $K$-valuation that assigns to each variable in $X \in \operatorname{vars}\left(B_{A}\right)$ the value $X^{K}(i)$. Initially, $X^{K}(0)=\emptyset$ for all $X \in \operatorname{vars}\left(B_{A}\right)$. In all subsequent iterations, the value of each variable $X \in \operatorname{vars}\left(B_{A}\right)$ is updated according to the equation $X^{K}(i+1)=\operatorname{expand}(X)^{K}\left(\Gamma^{K}(i)\right)$. Since the modal expressions in $B_{A}$ are monotonic, once $X_{F}^{K}(m) \neq \emptyset$ for some $m$, we know that

\footnotetext{
${ }^{2}$ Recall that we work, for convenience, in a setting that is dual to the one that considers linear properties to consist of all non-error traces.
} 
$X_{F}^{K}(n) \neq \emptyset$ for all $n>m$. Hence, we can detect that $K \models \theta_{A}$ as soon as $X_{F}^{K}(m)$ is nonempty. The following theorem guarantees that if there is an error trace of length $m$, then we will find it in $m$ iterations. In other words, using symbolic forward state traversal, we will explore only states up to distance $m$ from initial states.

Theorem 1. For every safety automaton $A$, an equivalent alternation-free post- $\mu_{\emptyset}$ query $\theta_{A}$ can be constructed in linear time. Further, for every Kripke structure $K$, if the shortest error trace in $K$ with respect to $A$ has length $m$, then $X_{F}^{K}(m) \neq \emptyset$, where $X_{F}$ is the root variable of $\theta_{A}$.

Büchi automata Safety automata cannot specify infi nite error traces. For that, we use Büchi automata. A Büchi automaton $A$ is a fi nite automaton such that a path $\pi$ of $A$ is accepting if $\operatorname{Inf}(\pi) \cap S_{F} \neq \emptyset$; that is, some accepting state of $A$ occurs infi nitely often in $\pi$. It is well-known [EL86,Dam94,BC96] that for every Büchi automaton $A$, there exists a $p r e-\mu_{\emptyset}$ query $\vartheta_{A}$ such that for every Kripke structure $K$, we have $K \models \exists A$ iff $K \models \vartheta_{A}$. We now show that there exists also a post- $\mu_{\emptyset}$ query $\theta_{A}$ with the same property, thereby proving that the model-checking problem for Büchi automata lies in the intersection of pre- $\mu_{\emptyset}$ and post- $\mu_{\emptyset}$. We defi ne two equational blocks: a $\nu$-block $B_{1}$ and a $\mu$-block $B_{2}$. The block $B_{1}$ contains the following $\left|S_{F}\right|+1$ equations, with $\operatorname{vars}\left(B_{1}\right)=\left\{X_{f}^{\prime} \mid f \in S_{F}\right\} \cup\left\{X_{F}^{\prime}\right\}$ :

$$
\begin{aligned}
& X_{f}^{\prime}=X_{f} \wedge \bigvee_{t \in \text { pre }(f)} \exists \odot X_{t}^{\prime}, \\
& X_{F}^{\prime}=\bigvee_{f \in S_{F}} X_{f}^{\prime} .
\end{aligned}
$$

The block $B_{2}$ contains an equation for each state $s \in S \backslash S_{F}$, defi ned by

$$
X_{s}^{\prime}=\gamma_{s} \wedge \bigvee_{t \in \text { pre }(s)} \exists \odot X_{t}^{\prime}
$$

Then, $\theta_{A}=\neg \mathcal{E}\left(\left\langle\left\langle B_{1}, B_{2}, B_{A}\right\rangle, X_{F}^{\prime}\right\rangle\right)$. Notice that, as with pre- $\mu_{\emptyset}$ [BC96], the translation is linear in the size of the Büchi automaton. Also, the equational blocks $B_{1}$ and $B_{2}$ depend on each other and the alternation depth of $\theta_{A}$ is two. Since Büchi automata are expressively equivalent to the $\omega$-regular languages, the query logic post- $\mu_{\emptyset}$ can specify all $\omega$-regular properties.

Theorem 2. For every Büchi automaton, an equivalent post- $\mu_{\emptyset}$ query of alternation depth two can be constructed in linear time.

In particular, since all sentences of the linear temporal logic LTL can be translated to Büchi automata [VW94], Theorem 2, together with [EL86], implies that all LTL sentences lie in the intersection pre- $\mu_{\emptyset} \cap$ post $-\mu_{\emptyset}$. Hence, LTL model checking can proceed by symbolic forward state traversal. Since the translation from LTL to Büchi automata involves an exponential blowup, the translation from LTL to post- $\mu_{\emptyset}$ is also exponential.

Co-Büchi automata Recall that the translation from Theorem 2 results in formulas of alternation depth two. It has been recently argued [KV98] that a linear property given by a co-Büchi automaton can be translated into an alternation-free pre- $\mu_{\emptyset}$ query. ${ }^{3}$ Consequently, the model checking of linear properties that are specifi ed by co-Büchi automata requires time that is only linear in the size of the Kripke structure. We now show that every co-Büchi automaton $A$ can also be translated into an equivalent alternation-free post- $\mu_{\emptyset}$ query $\theta_{A}$, thereby proving that the model-checking problem for co-Büchi automata lies in the intersection of alternation-free $p r e-\mu_{\emptyset}$ and alternation-free post- $\mu_{\emptyset}$. A co-Büchi automaton $A$ is a fi nite automaton such that a path $\pi$ of

\footnotetext{
${ }^{3}$ The results in [KV98] refer to sentences of the form $\forall A$, for deterministic B üchi automata $A$. Since an $\omega$ regular language can be specified by a deterministic $\mathrm{B}$ üchi automaton iff its complement can be specified by a co-B uchi automaton, the corresponding result for $\exists A$, for co-B üchi automata $A$, follows by duality.
} 
$A$ is accepting if $\operatorname{Inf}(\pi) \subseteq S_{F}$; that is, all the non-accepting states of $A$ occur in $\pi$ only fi nitely often. We defi ne an equational $\nu$-block $B_{3}$ that contains the following $\left|S_{F}\right|+1$ equations, with $\operatorname{vars}\left(B_{3}\right)=\left\{X_{f}^{\prime} \mid f \in S_{F}\right\} \cup\left\{X_{F}^{\prime}\right\}:$

$$
\begin{aligned}
& X_{f}^{\prime}=X_{f} \wedge \bigvee_{t \in \operatorname{pre}(f) \cap S_{F}} \exists \odot X_{t}^{\prime}, \\
& X_{F}^{\prime}=\bigvee_{f \in S_{F}} X_{f}^{\prime}
\end{aligned}
$$

Then, $\theta_{A}=\neg \mathcal{E}\left(\left\langle\left\langle B_{3}, B_{A}\right\rangle, X_{F}^{\prime}\right\rangle\right)$. Notice that $\theta_{A}$ is alternation-free and linear in the size of $A$.

Theorem 3. For every co-Büchi automaton, an equivalent alternation-free post- $\mu_{\emptyset}$ query can be constructed in linear time.

\subsection{Out}

We now show that there exist branching temporal-logic specifications that cannot be model checked by evaluating post- $\mu_{\mathcal{E}}$ queries. A post- $\mu_{\mathcal{E}}$ query $\theta$ is equivalent to a pre- $\mu$ sentence $\phi$ if for every Kripke structure $K$, we have $K \models \phi$ iff $K \models \theta$. Consider the pre- $\mu$ sentence $\phi_{1}=\langle\langle\langle\nu,\{X=\exists \bigcirc p \wedge \exists \bigcirc X\}\rangle\rangle, X\rangle$, which is equivalent to the CTL sentence $\exists \square \exists \bigcirc p$, and consider the Kripke structures $K_{1}$ and $K_{1}^{\prime}$ appearing in Figure 1. It is easy to see that

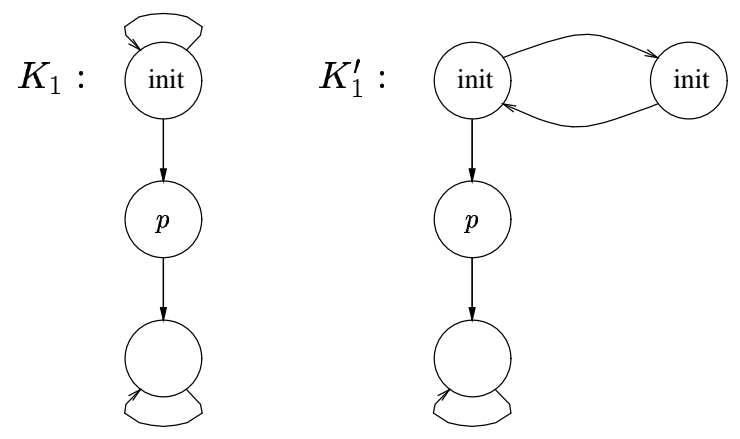

Fig. 1. $K_{1}$ and $K_{1}^{\prime}$ are post-bisimilar but not pre-bisimilar.

there is a post-bisimulation between $K_{1}$ and $K_{1}^{\prime}$. Hence, by Proposition 1, no post- $\mu_{\mathcal{E}}$ query can distinguish between them. On the other hand, while the structure $K_{1}$ satisfi es $\phi_{1}$, the structure $K_{1}^{\prime}$ does not satisfy $\phi_{1}$. Using a similar argument, it can be shown that the pre- $\mu$ sentence that is equivalent to the CTL sentence $\exists \diamond(r \wedge \exists \diamond p \wedge \exists \diamond q)$ can distinguish between two structures that have a post-bisimulation between them, implying there is no equivalent post- $\mu_{\mathcal{E}}$ query. Interestingly, the pre- $\mu$ sentence $\left\langle\left\langle B_{1}, B_{2}\right\rangle, X_{1}\right\rangle$ with $B_{1}=\left\langle\nu,\left\{X_{1}=X_{2} \wedge \exists \bigcirc X_{1}\right\}\right\rangle$ and $B_{2}=\left\langle\mu,\left\{X_{2}=p \vee \exists \bigcirc X_{2}\right\}\right\rangle$, which is equivalent to the CTL sentence $\exists \square \exists \diamond p$, and which is not equivalent to any LTL sentence [CD88], does have an equivalent query in post- $\mu_{\emptyset}$. The query is $\neg \mathcal{E}\left(\left\langle\left\langle B_{3}, B_{4}\right\rangle, X_{1}\right\rangle\right)$, with $B_{3}=\left\langle\nu,\left\{X_{1}=p \wedge X_{2}, X_{2}=X_{3} \wedge \exists \odot X_{2}\right\}\right\rangle$ and $B_{4}=\left\langle\mu,\left\{X_{3}=\right.\right.$ init $\left.\left.\vee \exists \odot X_{3}\right\}\right\rangle$.

Proposition 2. There exist pre- $\mu$ sentences (in fact, CTL sentences) that have no equivalent post- $\mu_{\mathcal{E}}$ queries.

\section{Hierarchy of Pre and Post Logics}

Let $\mathcal{L}_{1}$ and $\mathcal{L}_{2}$ be two logics whose sentences are interpreted over Kripke structures. The logic $\mathcal{L}_{2}$ is as expressive as the logic $\mathcal{L}_{1}$ if for every sentence $\phi_{1}$ of $\mathcal{L}_{1}$, there is a sentence $\phi_{2} \in \mathcal{L}_{2}$ 
such that for every Kripke structure $K$, we have $K \models \phi_{1}$ iff $K \models \phi_{2}$. The logic $\mathcal{L}_{2}$ is more expressive than $\mathcal{L}_{1}$ if $\mathcal{L}_{2}$ is as expressive as $\mathcal{L}_{1}$ but $\mathcal{L}_{1}$ is not as expressive as $\mathcal{L}_{2}$. The logic $\mathcal{L}_{2}$ is as distinguishing as the logic $\mathcal{L}_{1}$ if for all Kripke structures $K$ and $K^{\prime}$, if there is a sentence $\phi_{1}$ of $\mathcal{L}_{1}$ such that $K \models \phi_{1}$ but $K^{\prime} \not \models \phi_{1}$, then there is a sentence $\phi_{2}$ of $\mathcal{L}_{2}$ such that $K \models \phi_{2}$ but $K^{\prime} \not \models \phi_{2}$. Finally, the logic $\mathcal{L}_{2}$ is more distinguishing than $\mathcal{L}_{1}$ if $\mathcal{L}_{2}$ is as distinguishing as $\mathcal{L}_{1}$ but $\mathcal{L}_{1}$ is not as distinguishing as $\mathcal{L}_{2}$. In this section, we study the distinguishing and the expressive powers of $p r e-\mu$ and post- $\mu$ and the query logics they induce. For this purpose, the sentences of query logics are the queries.
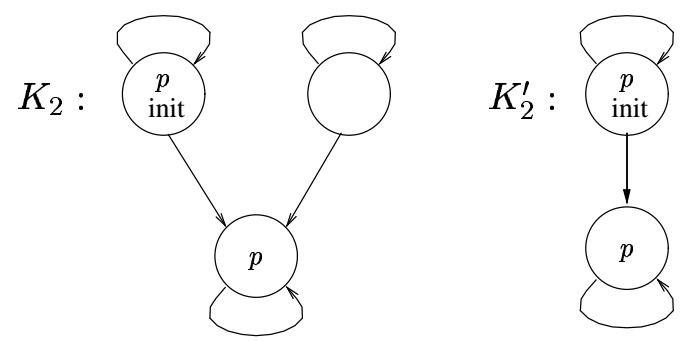

Fig. 2. $K_{2}$ and $K_{2}^{\prime}$ are init-post-bisimilar but not post-bisimilar

Proposition 3. The distinguishing powers of pre and post logics are summarized in the figure below. An arrow from logic $\mathcal{L}_{1}$ to logic $\mathcal{L}_{2}$ indicates that $\mathcal{L}_{1}$ is as distinguishing as $\mathcal{L}_{2}$. A line without arrow indicates incomparability.

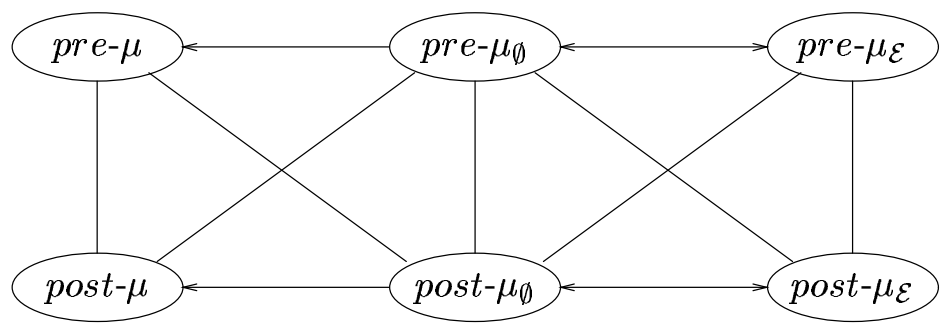

Proof. Proposition 1 implies that the distinguishing powers of pre- $\mu_{\emptyset}$ and $p r e-\mu_{\mathcal{E}}$ coincide, and similarly for post- $\mu$. In order to prove the incomparability results, we show that the four relations init-pre-bisimulation, init-post-bisimulation, pre-bisimulation, and post-bisimulation are all distinct. Recall that there may be states in a Kripke structure that are not reachable from an initial state, as there may be states from which no initial state is reachable. Consider the Kripke structures $K_{2}$ and $K_{2}^{\prime}$ appearing in Figure 2. There is an init-pre-bisimulation and an init-postbisimulation between $K_{2}$ and $K_{2}^{\prime}$, but no pre-bisimulation or post-bisimulation. Hence, (post) pre-bisimulation is more distinguishing than (init-post) init-pre-bisimulation. Now consider the Kripke structures $K_{1}$ and $K_{1}^{\prime}$ appearing in Figure 1. There is a post-bisimulation and an initpost-bisimulation between $K_{1}$ and $K_{1}^{\prime}$, but no pre-bisimulation or init-pre-bisimulation. Also, there is a pre-bisimulation and an init-pre-bisimulation between $K_{1}^{-1}$ and $K_{1}^{\prime-1}$ but no postbisimulation or init-post-bisimulation. Hence, pre-bisimulation and post-bisimulation as well as init-pre-bisimulation and init-post-bisimulation are incomparable.

Proposition 4. The expressive powers of pre and post logics are summarized in the figure below. An arrow from logic $\mathcal{L}_{1}$ to logic $\mathcal{L}_{2}$ indicates that $\mathcal{L}_{1}$ is as expressive as $\mathcal{L}_{2}$. A line without arrow indicates incomparability. 


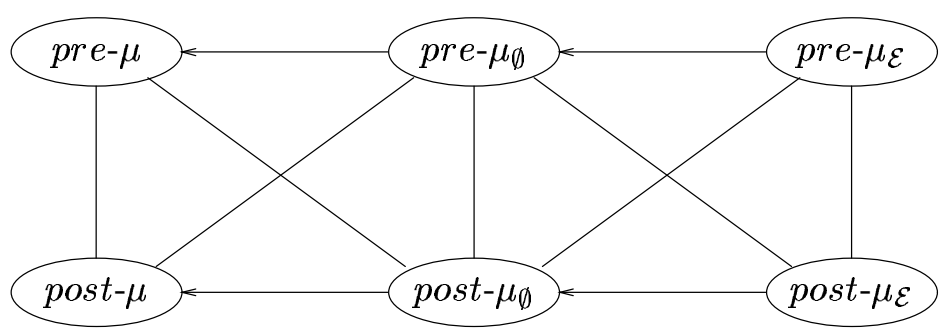

Proof. It is easy to see that if a logic $\mathcal{L}_{2}$ is not as distinguishing as a logic $\mathcal{L}_{1}$, then $\mathcal{L}_{2}$ is not as expressive as $\mathcal{L}_{1}$. Therefore, most of our expressiveness results follow from the corresponding results about distinguishability. In addition, as a Kripke structure $K$ satisfi es a sentence $\phi$ iff $K$ satisfi es the query $\neg \mathcal{E}($ init $\wedge \phi)$, the query logics pre- $\varphi_{\emptyset}$ and post- $\mu_{\emptyset}$ are more expressive than pre- $\mu$ and post- $\mu$, respectively. In order to prove the advantage of the full query logics pre- $\mu_{\mathcal{E}}$ and post- $\mu_{\mathcal{E}}$ over its subsets pre- $\mu_{\emptyset}$ and post- $\mu_{\emptyset}$, it is easy to see that no query of the query logics pre- $\mu_{\emptyset}$ and post- $\mu_{\emptyset}$ is equivalent to the query $\mathcal{E}(p) \vee \mathcal{E}(q)$.

\section{Discussion and Experimental Results}

\subsection{Intersection of pre and post logics}

While previous works presented symbolic forward state-traversal procedures for model checking some isolated linear and branching properties [INH96,IN97], we attempted to study more systematically the class of properties that can be model checked using both symbolic forward and backward state traversal. In particular, we showed that all $\omega$-regular linear properties (which includes all properties expressible in LTL) fall into this class, while some simple branching properties (expressible in CTL) do not. Furthermore, every query that can be specifi ed in both pre- $\mu_{\emptyset}$ and post- $\mu_{\emptyset}$ cannot distinguish between structures that are both pre-bisimilar and post-bisimilar. Yet the exact characterization of the intersection pre- $\mu_{\emptyset} \cap$ post- $\mu_{\emptyset}$ remains open. In [GK94], the authors identifi ed a set of temporal-logic sentences called equi-linear. In particular, a pre- $\mu$ sentence is equi-linear if it cannot distinguish between two Kripke structures with the same language (i.e., observation sequences that correspond to initialized paths). Clearly, all LTL sentences are equi-linear. However, some CTL sentences that have no equivalent LTL sentence are also equi-linear. For example, it is shown in [GK94] that while the CTL sentence $\exists \square \exists \bigcirc p$ is not equi-linear, the CTL sentence $\exists \square \exists \diamond p$ is equi-linear. Motivated by the examples from Section 3.2, we conjecture that equi-linearity precisely characterizes the properties that can be model checked using both symbolic forward and backward state traversal. Formally, we conjecture that a pre- $\mu$ sentence is equi-linear iff there is an equivalent post $-\mu_{\emptyset}$ query.

\subsection{Union of pre and post logics}

In this paper, we primarily think of post- $\mu_{\mathcal{E}}$ as a language for describing symbolic modelchecking procedures for temporal-logic specifi cations. Furthermore, we have focused on specifi cation languages that contain only future temporal operators. Since LTL with past temporal operators is no more expressive than LTL without past operators [LPZ85], every LTL+past sentence can also be translated into an equivalent post- $\mu_{\emptyset}$ query. In addition, post- $\mu$ also permits the easy evaluation of branching past temporal operators that cannot be evaluated using pre- $\mu$. For example, the sentence $\forall \square($ grant $\rightarrow(\neg$ init $) \forall \widetilde{\mathcal{W}}$ req $)$, where $\widetilde{\mathcal{W}}$ is a past version of the "weakuntil" operator [MP92], specifi es that grants are given only upon request. Assuming a branching interpretation for past temporal operators [KP95], this sentence has an equivalent post- $\mu_{\emptyset}$ query, but no equivalent pre- $\mu_{\mathcal{E}}$ query; that is, it can be model checked by symbolic forward state traversal but not by symbolic backward state traversal. 
While the intersection pre- $\mu_{\mathcal{E}} \cap$ post- $\mu_{\mathcal{E}}$ identifi es the queries that can be model checked by both symbolic forward and backward state traversal, it is the "union" (pre- $\mu \cup$ post- $\mu)_{\mathcal{E}}{ }^{4}$ that identifi es the queries that can be model checked at all symbolically, by mixed forward and backward state traversal. ${ }^{5}$ Furthermore, it is the alternation-free fragment of $(\text { pre- } \mu \cup \text { post- } \mu)_{\mathcal{E}}$ that identifi es the queries that can be model checked efficiently. Thus it is also of interest to ask which temporal logics can be translated into the (alternation-free) union of pre and post query logics. Such temporal logics can have both future and past temporal operators. In particular, it is easy to see that every CTL+past sentence (under the branching interpretation for past) has an equivalent query in the alternation-free fragment of $(\text { pre- } \mu \cup \text { post }-\mu)_{\emptyset}$.

\subsection{Experimental results}

In our experiments, we performed BDD-based symbolic model checking on a parameterized sliding-window protocol for the reliable transmission of packets over an unreliable channel. The parameter to the protocol is WINSIZE, the number of outstanding unacknowledged messages at the sender end. In the protocol, the messages are modeled as boolean values. We checked whether all computations of the protocol satisfy the partial specifi cation $\phi$, which states that if the produced message $m s g P$ toggles infi nitely often at the sender end, then so does the consumed message $m s g C$ at the receiver end. Formally, the specifi cation $\phi$ is given by the LTL sentence $\square \diamond(m s g P \leftrightarrow \bigcirc \neg m s g P) \rightarrow \square \diamond(m s g C \leftrightarrow \bigcirc \neg m s g C)$. We note that this sentence cannot be handled by the methods presented in [INH96,IN97].

In the table below we list the running times (in seconds) for different values of WINSIZE for checking $\phi$ using VIS [BHSV ${ }^{+}$96] for both symbolic forward and backward state traversal. The quantity within the parentheses is the number of boolean variables used to encode the state space of the protocol. It is folk wisdom in symbolic model checking that using don't-care minimization based on unreachable states can dramatically improve the running times. So we also applied fi rst symbolic forward state traversal to compute the set of reachable states and then symbolic backward state traversal for model checking, using the unreachable states as don't cares. These results are shown in the last column. A dash indicates an unsuccessful verifi cation attempt. In the future, we hope to compare our approach also against enumerative forward state-traversal methods for LTL model checking.

\begin{tabular}{|c|c|c|c|}
\hline WINSIZE & Forward & Backward & Reach-optimized backward \\
\hline $2(30)$ & 18 & 222 & 91 \\
$3(45)$ & 300 & 4584 & - \\
$4(50)$ & 5231 & - & - \\
\hline
\end{tabular}

\section{Acknowledgments}

We thank Rajeev Alur, Bob Brayton, Ed Clarke, Allen Emerson, and Orna Grumberg for helpful discussions, and Carl Pixley for drawing the authors' attention to [INH96].

\section{References}

[BC96] G. Bhat and R. Cleavland. Efficient model checking via the equational $\mu$-calculus. In Proc. 11th IEEE Symposium on Logic in Computer Science, pp. 304-312, 1996.

\footnotetext{
${ }^{4}$ By the union pre- $\mu \cup$ post- $\mu$ we refer to the logic with all four modal operators $\exists \bigcirc, \forall \bigcirc, \exists \odot$, and $\forall \odot$. It has, of course, strictly more sentences than the union of the sets of pre- $\mu$ and post- $\mu$ sentences.

${ }^{5}$ In fact, not only can model checking algorithms be extended from pre- $\mu$ to $(\text { pre- } \mu \cup \text { post- } \mu)_{\mathcal{E}}$ without extra cost, the satisfiability problem for the union is also no harder than the satisfiability problem for either pre- $\mu$ or post- $\mu$ [Var98].
} 
[BCG88] M.C. Browne, E.M. Clarke, and O. Grumberg. Characterizing finite Kripke structures in propositional temporal logic. Theoretical Computer Science, 59:115-131, 1988.

$\left[\mathrm{BCM}^{+}\right.$92] J.R. Burch, E.M. Clarke, K.L. McMillan, D.L. Dill, and L.J. Hwang. Symbolic model checking: $10^{20}$ states and beyond. Information and Computation, 98(2):142-170, 1992.

[BHSV ${ }^{+}$96] R.K. Brayton, G.D. Hachtel, A. Sangiovanni-Vincentelli, F. Somenzi, A. Aziz, S.-T. Cheng, S. Edwards, S. Khatri, T. Kukimoto, A. Pardo, S. Qadeer, R.K. Ranjan, S. Sarwary, T.R. Shiple, G. Swamy, and T. Villa. VIS: a system for verification and synthesis. In CAV 96: Computer Aided Verifi cation, LNCS 1102, pp. 428-432, Springer, 1996.

[Bry86] R.E. Bryant. Graph-based algorithms for boolean-function manipulation. IEEE Trans. on Computers, C-35(8), 1986.

[CD88] E.M. Clarke and I.A. Draghicescu. Expressibility results for linear-time and branching-time logics. In Proc. Workshop on Linear Time, Branching Time, and Partial Order in Logics and Models for Concurrency, LNCS 354, pp. 428-437, Springer, 1988.

[CE81] E.M. Clarke and E.A. Emerson. Design and synthesis of synchronization skeletons using branching time temporal logic. In Proc. Workshop on Logic of Programs, LNCS 131, pp. 52-71, Springer, 1981.

[CGH94] E.M. Clarke, O. Grumberg, and K. Hamaguchi. Another look at LTL model checking. In $C A V$ 94: Computer Aided Verifi cation, LNCS 818, pp. 415 - 427, Springer, 1994.

[CGL93] E.M. Clarke, O. Grumberg, and D. Long. Verification tools for finite-state concurrent systems. In Decade of Concurrency - Reflections and Perspectives (Proc. REX School), LNCS 803, pp. 124-175, Springer, 1993.

[CS91] R. Cleaveland and B. Steffen. A linear-time model-checking algorithm for the alternation-free modal $\mu$-calculus. In CAV 91: Computer Aided Verifi cation, LNCS 575, pp. 48-58, Springer, 1991.

[Dam94] M. Dam. CTL ${ }^{\star}$ and $\mathrm{ECTL}^{\star}$ as fragments of the modal $\mu$-calculus. Theoretical Computer Science, 126:77-96, 1994.

[Di196] David L. Dill. The Mur $\phi$ Verification System. In CAV 96: Computer Aided Verifi cation, LNCS 1102, pp. 390-393, Springer, 1996.

[EL86] E.A. Emerson and C.-L. Lei. Efficient model checking in fragments of the propositional $\mu$-calculus. In Proc. 1st Symposium on Logic in Computer Science, pp. 267-278, 1986.

[GK94] O. Grumberg and R.P. Kurshan. How linear can branching-time be. In Proc. 1st International Conference on Temporal Logic, LNAI 827, pp. 180-194, Springer, 1994.

[GPVW95] R. Gerth, D. Peled, M.Y. Vardi, and P. Wolper. Simple on-the-fly automatic verification of linear temporal logic. In Protocol Specifi cation, Testing, and Verifi cation, pp. 3-18, Chapman, 1995.

[Hol97] G.J. Holzmann. The model checker SPIN. IEEE Trans. on Software Engineering, 23(5):279-295, 1997.

[IN97] H. Iwashita and T. Nakata. Forward model checking techniques oriented to buggy designs. In Proc. IEEE/ACM International Conference on Computer Aided Design, pp. 400-404, 1997.

[INH96] H. Iwashita, T. Nakata, and F. Hirose. CTL model checking based on forward state traversal. In Proc. IEEE/ACM International Conference on Computer Aided Design, pp. 82-87, 1996.

[Koz83] D. Kozen. Results on the propositional $\mu$-calculus. Theoretical Computer Science, 27:333-354, 1983.

[KP95] O. Kupferman and A. Pnueli. Once and for all. In Proc. 10th IEEE Symposium on Logic in Computer Science, pp. 25-35, 1995.

[KV98] O. Kupferman and M.Y. Vardi. Freedom, weakness, and determinism: from linear-time to branching-time. In Proc. 13th IEEE Symposium on Logic in Computer Science, 1998.

[LPZ85] O. Lichtenstein, A. Pnueli, and L. Zuck. The glory of the past. In Logics of Programs, LNCS 193, pp. 196-218, Springer, 1985.

[McM93] K.L. McMillan. Symbolic Model Checking. Kluwer Academic Publishers, 1993.

[MP92] Z. Manna and A. Pnueli. The Temporal Logic of Reactive and Concurrent Systems: Specifi cation. Springer, 1992.

[Pe194] D. Peled. Combining partial order reductions with on-the-fly model-checking. In CAV 94: Computer Aided Verifi cation, LNCS 818, pp. 377-390, Springer, 1994.

[QS81] J.P. Queille and J. Sifakis. Specification and verification of concurrent systems in Cesar. In Proc. 5th International Symp. on Programming, LNCS 137, pp. 337-351, Springer, 1981.

[Var98] M.Y. Vardi. Reasoning about the past with two-way automata. In Proc. 25th International Coll. on Automata, Languages, and Programming, LNCS, Springer, 1998.

[VW94] M.Y. Vardi and P. Wolper. Reasoning about infinite computations. Information and Computation, 115(1):1-37, 1994. 